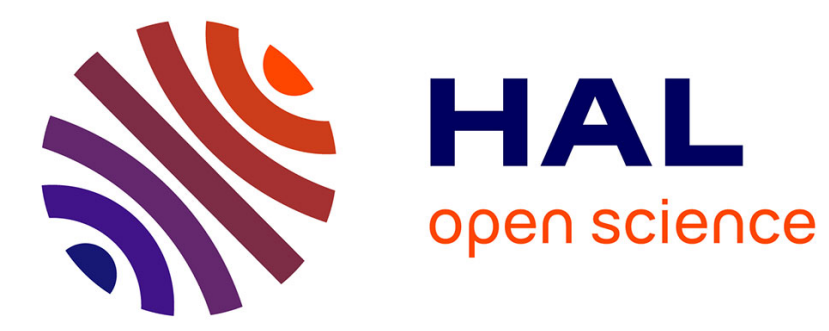

\title{
Le chrome noir et la sélectivité aux moyennes températures $\left(100-250{ }^{\circ} \mathrm{C}\right)$
}

\author{
J. Lafait, J.M. Behaghel, Serge Berthier
}

\section{To cite this version:}

J. Lafait, J.M. Behaghel, Serge Berthier. Le chrome noir et la sélectivité aux moyennes températures (100-250 $\left.{ }^{\circ} \mathrm{C}\right)$. Revue de Physique Appliquée, 1980, 15 (3), pp.403-409. 10.1051/rphysap:01980001503040300 . jpa-00244744

\section{HAL Id: jpa-00244744 https://hal.science/jpa-00244744}

Submitted on 1 Jan 1980

HAL is a multi-disciplinary open access archive for the deposit and dissemination of scientific research documents, whether they are published or not. The documents may come from teaching and research institutions in France or abroad, or from public or private research centers.
L'archive ouverte pluridisciplinaire HAL, est destinée au dépôt et à la diffusion de documents scientifiques de niveau recherche, publiés ou non, émanant des établissements d'enseignement et de recherche français ou étrangers, des laboratoires publics ou privés. 


\title{
Le chrome noir et la sélectivité aux moyennes températures $\left(100-250{ }^{\circ} \mathrm{C}\right)$
}

\author{
J. Lafait, J. M. Behaghel et S. Berthier \\ Laboratoire d’Optique des Solides (*), Université Pierre-et-Marie-Curie, 4, place Jussieu, 75230 Paris Cedex 05, France
}

(Reçu le 4 août 1979, révisé le 21 novembre 1979, accepté le 26 novembre 1979)

\begin{abstract}
Résumé. - Les capteurs photothermiques travaillant sous faible ou moyenne concentration $(<100)$ sont en plein développement. On montre sur des exemples théoriques très proches de la réalité l'intérêt des surfaces sélectives associées à de tels capteurs. On présente enfin en détail les propriétés optiques des surfaces sélectives au Chrome Noir stables jusqu'à $300^{\circ} \mathrm{C}$. L'absorption solaire a de ces surfaces reste voisine de $95 \%$ jusqu'à $250^{\circ} \mathrm{C}$, le rapport absorption solaire/émission infrarouge (a/e) étant de l'ordre de 10 à cette température.
\end{abstract}

\begin{abstract}
Photothermal collectors working under low or middle concentration $(<100)$ are now strongly developing : One shows, by means of theoretical calculations very close to reality, the interest of selective surfaces associated with such collectors. Optical properties of Black Chromium selective surfaces, stable up to $300^{\circ} \mathrm{C}$, are then detailed. Solar absorptance a remains equal to $95 \%$ up to $250{ }^{\circ} \mathrm{C}$, solar absorptance/infrared emittance ratio $(\mathrm{a} / \mathrm{e})$ being around 10 at this temperature.
\end{abstract}

1. Introduction. - Les capteurs photothermiques à faible ou moyenne concentration $(X<100)$ utilisant comme système concentrateur du flux solaire un miroir cylindroparabolique, parabolique ou sphérique sont actuellement en plein développement. Les systèmes à moyenne concentration sont généralement équipés de servomécanismes déplaçant le miroir ou l'absorbeur ou les deux éléments à la fois, afin de toujours focaliser le maximum du flux solaire direct sur l'absorbeur. Dans ces conditions, des températures d'absorbeur de plus de $500{ }^{\circ} \mathrm{C}$ peuvent être atteintes. Ces systèmes constituent le maillon intermédiaire entre les capteurs plans et les centrales à tour utilisant un champ complet de miroirs de focalisation. On a déjà montré [1] que les surfaces absorbantes optiquement sélectives permettaient une augmentation appréciable du rendement de ces capteurs pour des facteurs de concentration inférieurs à 100 et des températures d'absorbeur inférieures à $700{ }^{\circ} \mathrm{C}$ environ.

Le présent travail concerne plus particulièrement les capteurs à faible concentration $(X$ de l'ordre de 10 à 20) ou à plus forte concentration, mais devant présenter un bon rendement même aux faibles flux solaires. Les températures atteintes par les absorbeurs de ces capteurs ne dépassent guère $250^{\circ} \mathrm{C}$. Leur domaine d'application recouvre le chauffage des

$\left(^{*}\right)$ Equipe de Recherche Associée no 462. habitations, la réfrigération, la climatisation, le déssalement de l'eau de mer, la production d'énergie mécanique et de vapeur, les usages de l'industrie agroalimentaire, une partie de la chimie du bois...

Dans une première partie nous présentons quelques calculs simples portant sur le rendement des capteurs sans vitrage et permettant d'évaluer le gain apporté par l'utilisation d'un revêtement sélectif sur l'absorbeur en fonction du flux incident, du facteur de concentration et de la température de l'absorbeur. Le profil spectral de sélectivité utilisé dans ces calculs est aussi proche que possible du profil réel des matériaux sélectifs connus et permet ainsi une évaluation du gain bien plus précise que les profils théoriques en marche d'escalier généralement utilisés qui surestiment l'absorption solaire et sousestiment l'émission infrarouge.

Dans une seconde partie, nous présentons en détail les propriétés optiques sélectives du chrome noir, préparé au L.E.M.M. (C.E.N.G.), particulièrement bien adapté à ce domaine des températures moyennes.

Le chrome noir présente une excellente stabilité jusqu'à $300^{\circ} \mathrm{C}$ et ses propriétés optiques ne sont pratiquement pas modifiées jusqu'à $250^{\circ} \mathrm{C}$. Nous insistons particulièrement sur la diffusion et sur les variations des propriétés optiques en fonction de l'incidence en vue de l'utilisation sur des absorbeurs recevant le flux solaire sous des incidences non normales ou variables. 
2. Gain apporté par la sélectivité aux températures moyennes $\left(100-250{ }^{\circ} \mathrm{C}\right) .-2.1$ DÉFINITION DU GAIN. - Afin de comparer les performances de deux capteurs, nous allons partir d'une expression simplifiée du rendement de conversion :

$$
\eta=a-\frac{e}{X: E_{\mathrm{s}}} \cdot \sigma\left(T_{\mathrm{a}}^{4}-T_{0}^{4}\right)-K=\eta_{\mathrm{R}}-K
$$

où :

$a=$ absorptivité solaire de l'absorbeur,

$e=$ émissivité de l'absorbeur,

$E_{\mathrm{s}}=$ éclairement solaire direct au sol sur une surface normale au rayonnement (W. $\mathrm{m}^{-2}$ ),

$X=$ facteur de concentration du flux incident,

$T_{0}=$ température équivalente de l'environnement $(\mathrm{K})$,

$T_{\mathrm{a}}=$ température de l'absorbeur $(\mathrm{K})$,

$K=$ facteur traduisant l'effet des pertes optiques, conductives, convectives,

$\eta_{\mathbf{R}}=$ rendement radiatif.

L'émissivité $e$ de l'absorbeur est définie à partir de l'émissivité monochromatique $\varepsilon(\lambda, T)$ et de l'émittance spectrale $M_{0}(\lambda, T)$ du corps noir à la température $T$ par :

$$
e=\int_{0}^{\infty} \varepsilon(\lambda, T) M_{0}(\lambda, T) \mathrm{d} \lambda / \int_{0}^{\infty} M_{\iota}(\lambda, T) \mathrm{d} \lambda
$$

$\varepsilon(\lambda, T)$ résulte elle-même de l'intégration sur tout le demi-espace de l'émissivité monochromatique directionnelle $\varepsilon^{\prime}(\lambda, T, \theta, \varphi)$.

Il en est de même de l'absorptivité solaire $a$ définie à partir de l'absorptivité monochromatique intégrée $\alpha(\lambda, T)$ et de l'éclairement solaire direct $E_{\mathrm{s}}(\lambda)$ par

$$
a=\int_{0}^{\infty} \alpha(\lambda, T) E_{\mathrm{s}}(\lambda) \mathrm{d} \lambda / \int_{0}^{\infty} E_{\mathrm{s}}(\lambda) \mathrm{d} \lambda .
$$

Il est important de considérer qu'il s'agit là de valeurs moyennes car les propriétés optiques des surfaces absorbantes sont en général rapidement variables avec l'incidence (particulièrement entre 50 et $90^{\circ}$ ). Les capteurs sous concentration travaillant souvent à incidence variable ou avec un angle d'ouverture important sur l'absorbeur, une étude plus fine devrait envisager le calcul de $a$ à partir d'une intégration prenant en compte la géométrie propre à chaque système.

Dans l'expression (1), l'émissivité de l'absorbeur à sa température $T_{\mathrm{a}}$ est confondue avec son absorptivité à la température $T_{0}$ dans l'expression des pertes radiatives e. $\sigma\left(T_{\mathrm{a}}^{4}-T_{0}^{4}\right)$. Un calcul complet de l'erreur introduite par cette simplification montre que, dans les cas les plus défavorables correspondant à des conditions pratiques de fonctionnement, elle est toujours inférieure à $2,5 \%$. Enfin, l'émissivité $e$ a été définie comme étant celle de l'absorbeur, ce qui suppose que le capteur fonctionne sans vitrage de couverture. C'est le cas d'un grand nombre de capteurs travaillant dans ce domaine des concentrations moyen- nes. Remarquons que dans ces conditions deux capteurs fonctionnant à la même température $T_{\mathrm{a}}$ auront des pertes conductives et convectives identiques et que leurs facteurs $K$ (formule (1)) seront donc égaux.

Il est alors possible de comparer deux types de capteurs en étudiant leurs rendements radiatifs respectifs, sachant que le rendement de conversion global pourra être obtenu en retranchant la même quantité $K$ aux deux rendements radiatifs :

$$
\eta=a\left[1-\frac{e}{a} \cdot \frac{\sigma}{X . E_{\mathrm{s}}}\left(T_{\mathrm{a}}^{4}-T_{0}^{4}\right)\right]=a(1-P) .
$$

On remarque d'emblée que deux surfaces absorbantes, ayant la même absorption solaire $a$ et des émissivités différentes (ce qui est grossièrement le cas des surfaces sélectives et non sélectives), auront des performances d'autant plus différentes que le facteur $P$ sera grand, c'est-à-dire lorsque :

- la température de l'absorbeur sera élevée,

- ou la concentration faible,

- ou le flux solaire faible.

En d'autres termes et pour aborder le problème de la sélectivité, si avec une surface noire non sélective on obtient un rendement radiatif proche de 1 , le facteur $P$ est petit et l'utilisation d'une surface sélective présentant une valeur $e$ plus faible n'apportera pas un gain considérable. $\mathrm{Si}$, par contre, on est loin du rendement maximum ( $P$ grand), la sélectivité présentera un grand intérêt. Ce sont ces considérations qualitatives que nous allons développer maintenant d'une manière plus détaillée.

2.2 Comparaison SÉleCtif-NON SÉleCTIF. - Nous considérons des revêtements non sélectifs présentant une absorptivité monochromatique constante dans le spectre visible $\alpha(\lambda)=0,95$ et une émissivité monochromatique constante dans l'infrarouge $\varepsilon(\lambda)=0,90$ que nous supposerons en première approximation indépendantes de la température.

2.2.1 Influence de la longueur d'onde de coupure sur le rendement des surfaces sélectives théoriques et réelles. - Nous considérons des revêtements sélectifs présentant à température ambiante une absorptivité monochromatique constante dans le visible $\alpha(\lambda)=0,95$ et une émissivité monochromatique constante dans l'infrarouge $\varepsilon(\lambda)=0,05$. Le revêtement sélectif que nous qualifierons de théorique (généralement utilisé dans les calculs sur les absorbeurs sélectifs) présente une transition absorbant visible-réflecteur infrarouge verticale à la longueur d'onde $\lambda_{\mathrm{c}}$ (Fig. 1), tandis que le revêtement que nous qualifions de réel dans la mesure où ses propriétés optiques sont proches de celles des matériaux sélectifs connus présente une transition en pente (Fig. 2) suivant la loi :

$R=0,497 \log \frac{\lambda}{\lambda_{\mathrm{c}}}+0,5$ pour $0,05<R(\lambda)<0,95$. 


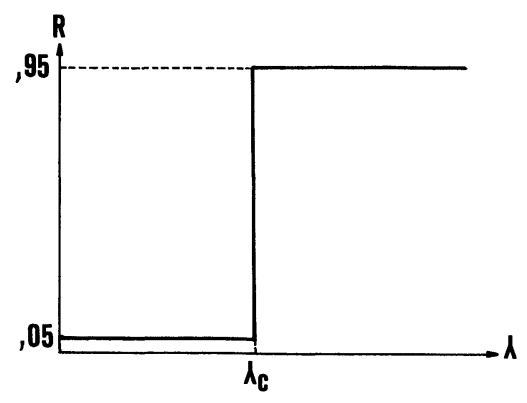

Fig. 1. - Spectre de réflectivité d'une surface sélective théorique.

[Spectral reflectivity of a theoretical selective surface.]

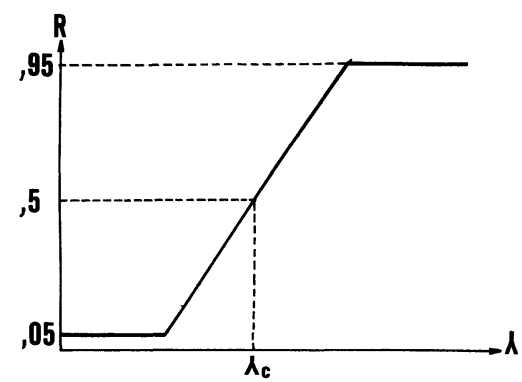

Fig. 2. - Spectre de réflectivité d'une surface sélective réelle.

[Spectral reflectivity of a real selective surface.]

Tableau I. - Absorption solaire (masse d'air 1) et émissivité d'une surface sélective réelle de l'ambiante à $300^{\circ} \mathrm{C}$ pour trois longueurs d'onde de coupure.

[Solar absorptance (air mass 1) and infrared emittance of a real selective surface from room temperature up to $300{ }^{\circ} \mathrm{C}$ for three cut-off wavelengths.]

\begin{tabular}{|c|c|c|c|c|c|c|c|c|}
\hline \multirow{2}{*}{$\lambda_{-}(\mu \mathrm{m})$} & \multirow[t]{2}{*}{$a(\%)$} & \multicolumn{7}{|c|}{$e(\%)$} \\
\hline & & $20^{\circ}$ & $50^{\circ}$ & $80^{\circ}$ & $110^{\circ}$ & $140^{\circ}$ & $200^{\circ}$ & $300^{\circ} \mathrm{C}$ \\
\hline 1,65 & 82 & 5,0 & 5,0 & 5,1 & 5,2 & 5,4 & 5,8 & 6,2 \\
\hline 2,70 & 91 & 5,6 & 6,0 & 6,5 & 7,1 & 7,9 & 9,9 & 13,5 \\
\hline 3,65 & 93,2 & 7,3 & 8,4 & 9,8 & 11,1 & 12,6 & 15,9 & 21,6 \\
\hline
\end{tabular}

Le tableau I fournit les valeurs de l'émissivité $e$ de la surface sélective réelle calculée en fonction de la température, de l'ambiante à $300^{\circ} \mathrm{C}$, pour trois longueurs d'onde de coupure : 1,$65 ; 2,70$ et $3,65 \mu \mathrm{m}$, ainsi que celles de l'absorption solaire a pour les mêmes $\lambda_{\mathrm{c}}$.

La figure 3 présente ces résultats sous forme de courbes auxquelles on a ajouté les valeurs correspondantes de la surface sélective théorique pour $\lambda_{\mathrm{c}}=2,25$ et $3 \mu \mathrm{m}$. On remarque des différences importantes entre ces deux cas. Ainsi pour $\lambda_{\mathrm{c}}=2,2 \mu \mathrm{m} a$ (sélectif théorique) $=0,93$ et $a$ (sélectif réel) $=0,87$. Pour $\lambda_{\mathrm{c}}=3,0 \mu \mathrm{m}, T=300^{\circ} \mathrm{C}, e($ sélectif théorique $)=0,07$, $e$ (sélectif réel) $=0,16$.

Nous utiliserons donc pour la suite des calculs le profil de sélectivité que nous avons appelé réel et qui est proche de la moyenne des résultats obtenus avec divers revêtements sélectifs développés pour ces températures moyennes.

Vers $300^{\circ} \mathrm{C}$ on voit clairement apparaître (Fig. 3) le compromis entre une émissivité modérée et une absorption solaire encore appréciable pour une longueur d'onde de coupure se situant aux alentours de $3 \mu \mathrm{m}$. Si $\lambda_{\mathrm{c}}$ baisse, $e$ diminue mais également $a$. Si $\lambda_{\text {c }}$ augmente, $a$ augmente mais aussi $e$.

2.2.2 Variations du rendement radiatif en fonction du flux solaire incident. - Nous comparons ici les rendements radiatifs des surfaces non sélective et sélective réelle définies au début du paragraphe 2 (avec $\alpha$ de la surface sélective $=0,96$ au lieu de 0,95 afin de mieux correspondre aux surfaces proches du chrome noir et $\lambda_{\mathrm{c}}=4 \mu \mathrm{m}$ ).

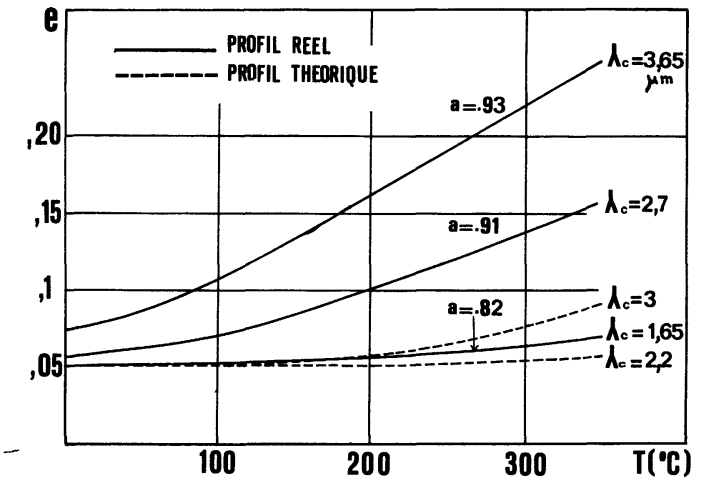

Fig. 3. - Emissivité de la surface sélective réelle et d'une surface sélective théorique en fonction de la température d'absorbeur pour diverses longueurs d'onde de coupure.

[Emittance of the real selective surface and of a non selective one versus absorber temperature for different cut-off wavelengths.]

La figure 4 présente ces rendements pour une température équivalente d'environnement $T_{0}$ fixée à $10^{\circ} \mathrm{C}$, la température de l'absorbeur $T_{\mathrm{a}}$ apparaissant comme paramètre à $100,150,200^{\circ} \mathrm{C}$. Dans la figure 5 au contraire, $T_{\mathrm{a}}$ est fixée à $150^{\circ} \mathrm{C}$ et $T_{0}$ varie entre -20 et $+40^{\circ} \mathrm{C}$. Le flux solaire incident $X . E_{\mathrm{s}}$ est compris entre 400 et $15000 \mathrm{~W} \cdot \mathrm{m}^{-2}$, ce qui nous situe dans des ensoleillements moyens avec concentration moyenne ou n'importe quelle combinaison fortfaible.

Comme nous l'avions remarqué qualitativement à la fin du paragraphe 1, le gain apporté par la sélectivité ne devient appréciable que lorsque le rendement de la surface non sélective est inférieur à $80 \%$. Par 


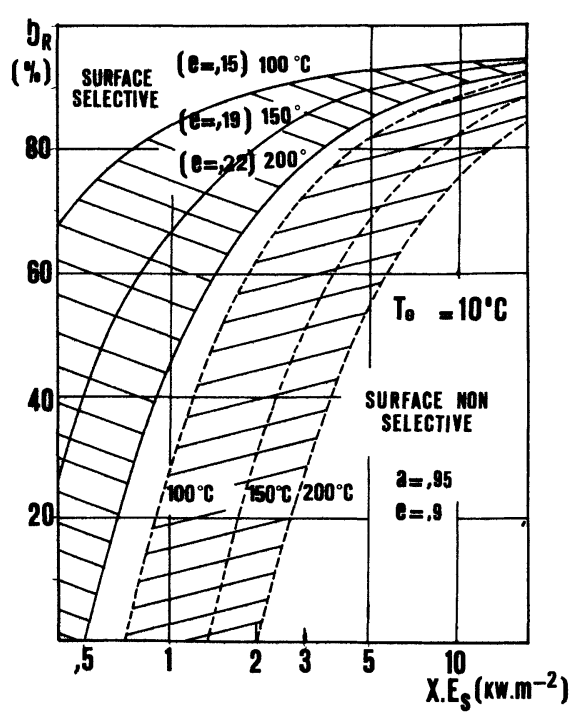

Fig. 4. - Rendement radiatif de la surface sélective réelle et d'une surface non sélective en fonction de l'énergie solaire incidente pour diverses températures d'absorbeur.

[Radiative efficiency of the real selective surface and of a non selective one versus incident solar flux for different absorber temperatures.]

contre, on peut voir (Fig. 5) qu'il est pratiquement impossible d'obtenir une température d'absorbeur de $150^{\circ} \mathrm{C}$ avec une surface non sélective si le flux incident est inférieur à $1800 \mathrm{~W} \cdot \mathrm{m}^{-2}\left(X=2, E_{\mathrm{s}}=900 \mathrm{~W} \cdot \mathrm{m}^{-2}\right.$ ou $X=20, E_{\mathrm{s}}=90 \mathrm{~W} \cdot \mathrm{m}^{-2}$ ), tandis qu'avec cette énergie le rendement de la surface sélective est de l'ordre de $80 \%$. Pour ce même flux incident, remarquons (Fig. 4) l'influence de la température sur l'émissivité de la surface, qui fait tomber le rendement radiatif de $89 \%$ à $100^{\circ} \mathrm{C}$ à $68 \%$ à $200{ }^{\circ} \mathrm{C}$. Notons

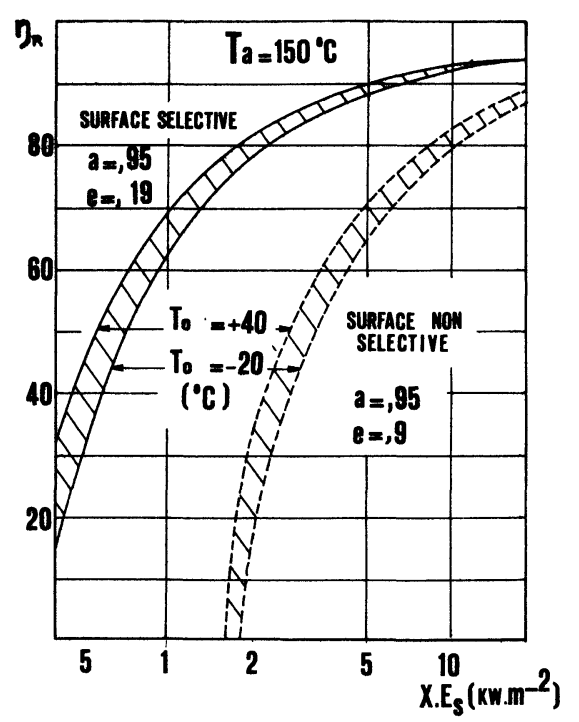

Fig. 5. - Rendement radiatif de la surface sélective réelle et d'une surface non sélective en fonction de l'énergie solaire incidente pour diverses températures équivalentes d'environnement.

[Radiative efficiency of the real selective surface and of a non selective one versus incident solar flux for different air temperatures.] enfin (Fig. 5) que pour des flux appréciables $\left(>2000 \mathrm{~W} \cdot \mathrm{m}^{-2}\right)$, l'influence de la température équivalente d'environnement sur le rendement est faible. Rappelons que nous exprimons là des rendements radiatifs : les pertes convectives et conductives ne sont pas prises en compte.

2.2.3 Gain énergétique entre absorbeurs sélectif et non sélectif. - Pour passer à des notions plus directement appréciables, nous avons représenté sur la figure 6 l'énergie disponible (pertes convectives et conductives non déduites) en fonction du flux incident $\left(T_{\mathrm{a}}=150^{\circ} \mathrm{C}, T_{0}=10^{\circ} \mathrm{C}\right)$. Il est clair d'après la formule (4) que sa variation est linéaire et que la prise en compte de la sélectivité (les absorptions solaires des deux surfaces étant égales) ne fait que translater la droite vers les énergies croissantes d'une quantité $G$ (dans ce cas $1100 \mathrm{~W} . \mathrm{m}^{-2}$ ), indépendante

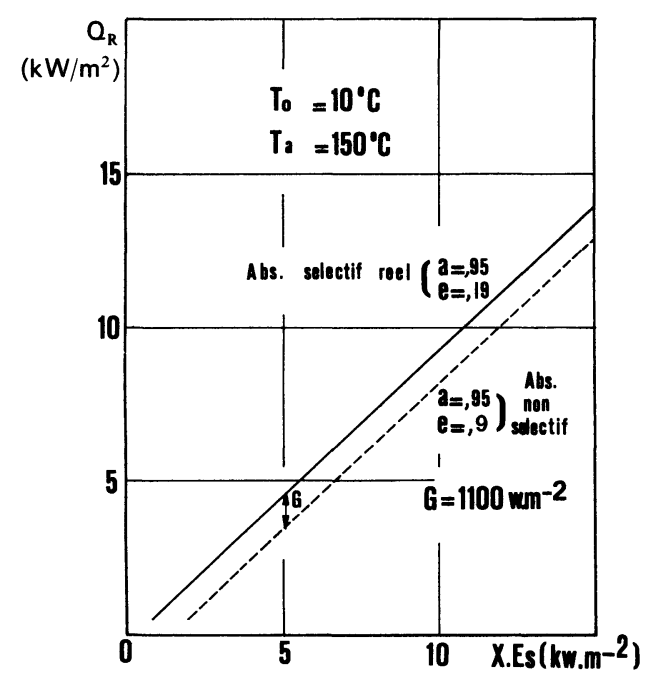

Fig. 6. - Energie disponible en fonction du flux solaire incident $\left(T_{\mathrm{a}}=150^{\circ} \mathrm{C}, T_{0}=10^{\circ} \mathrm{C}\right)$.

[Available energy as a function of incident solar flux $\left(T_{\mathrm{a}}=150^{\circ} \mathrm{C}\right.$, $\left.T_{0}=10^{\circ} \mathrm{C}\right)$.]

par conséquent de l'énergie incidente. C'est ce gain énergétique $G$ apporté par la sélectivité que nous avons porté sur la figure 7 en fonction de la température de l'absorbeur. Entre 100 et $200^{\circ} \mathrm{C}$ ce gain varie de 600 à $1700 \mathrm{~W} \cdot \mathrm{m}^{-2}$. Mais les gains absolus sont des quantités parfois fort trompeuses, il convient de bien vérifier à quels rendements ils correspondent (Fig. 4).

Notons que si les hypothèses conduisant à la formule (1) sont vérifiées, c'est-à-dire si les pertes convectives et conductives sont les mêmes pour les deux surfaces, le gain radiatif représenté sur la figure 7 sera égal au gain sur l'énergie totale réellement disponible.

Ces quelques courbes permettent une bonne évaluation du gain apporté par une surface sélective représentative des surfaces les plus couramment développées actuellement pour les applications aux moyennes températures. Examinons maintenant plus en 


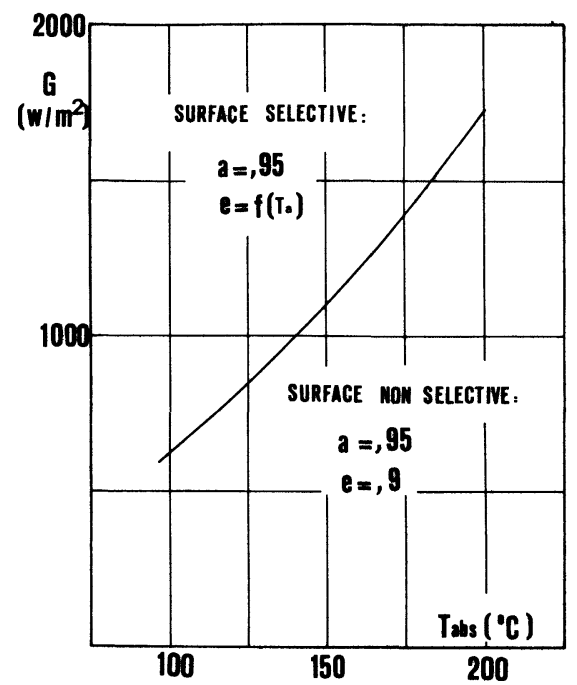

Fig. 7. - Gain énergétique entre un absorbeur sélectif réel et un absorbeur non sélectif en fonction de la température d'absorbeur.

[Difference between energy available in the case of a real selective absorber and a non selective one versus absorber temperature.]

détail les propriétés optiques des surfaces traitées au Chrome Noir.

3. Les surfaces sélectives au chrome noir. 3.1 Préparation et stabilité. - Il existe une grande variété de surfaces au Chrome Noir préparées par électrolyse. Cette variété s'explique par le nombre des paramètres de préparation dont les principaux sont la composition du bain, sa température, l'intensité du courant d'électrolyse, la durée du dépôt. Les propriétés optiques des principaux revêtements actuellement commercialisés pour être utilisés comme surfaces sélectives ne présentent pas de différences majeures : les valeurs de $a$ et $e$ (dans la mesure où l'on peut les comparer, compte tenu des techniques de caractérisation très diverses : photométriques, calorimétriques, directionnelles, hémisphériques...) ne diffèrent pas de plus de quelques pour-cents, seule la longueur d'onde de coupure varie entre 1,5 et $5 \mu \mathrm{m}$. Les différences, comme le montre une étude récente [2], portent essentiellement sur la structure du dépôt et se traduisent par une plus ou moins grande stabilité en fonction de la température. Le Chrome Noir dont nous présentons ici les propriétés a été mis au point au Laboratoire d'Etudes des Matériaux Minces du C.E.N.G., c'est le seul, à notre connaissance, à être actuellement commercialisé en France.

Les revêtements ont donc été préparés par électrolyse d'un bain à base d'acide chromique, exempt d'ions sulfates [3] à $25^{\circ} \mathrm{C}$ avec anode d'acier. On a utilisé comme substrat de l'acier nickelé, du cuivre nickelé et du cuivre nu. On a mis en évidence [4] un optimum des propriétés sélectives pour un temps de dépôt d'environ $90 \mathrm{~s}$ correspondant à un minimum de poids du revêtement [3]. Le dépôt optimal présente une structure composite sous forme de grains de $\mathrm{Cr}$ métallique disposés dans une matrice d'oxydes de chrome (sur $3000 \AA$ environ), l'ensemble étant recouvert d'une couche de $\mathrm{Cr}_{2} \mathrm{O}_{3}$ rugueuse d'environ $1000 \AA$. C'est cette structure inhomogène métal/oxydes de ce métal qui explique la lente dégradation des propriétés optiques du Chrome Noir au-dessus de $300^{\circ} \mathrm{C}$ par oxydation du métal et migration des particules. Jusqu'à $250^{\circ} \mathrm{C}$, par contre, les propriétés optiques sont remarquablement stables et le Chrome Noir présente même une résistance à l'abrasion, une stabilité aux cycles thermiques et une stabilité chimique incomparables avec les peintures absorbantes non sélectives couramment utilisées. C'est ainsi que l'on n'observe aucune dégradation des revêtements de Chrome Noir après 1450 cycles entre $60^{\circ}$ et $200^{\circ} \mathrm{C}$, alors que la peinture noire est totalement détériorée au bout de 100 cycles. De même, si le revêtement est soumis pendant $1200 \mathrm{~h}$ à un brouillard salin à $5 \%$ on n'observe aucune dégradation, alors qu'au bout de $50 \mathrm{~h}$ la peinture noire a été totalement éliminée par la rouille et les cloques.

3. 2 RÉFLECTIVITÉ HÉMISPHÉRIQUE. - La réflectivité hémisphérique des échantillons a été mesurée sous incidence voisine de la normale entre 0,5 et $15 \mu \mathrm{m}$ à l'aide d'un appareillage comprenant des sphères intégrantes originales décrit en détail ailleurs [5].

On présente les résultats relatifs à trois échantillons moyens caractéristiques des trois types étudiés : (1) dépôt sur acier nickelé, (2) dépôt sur cuivre avec bain amélioré, (3) dépôt sur cuivre nickelé avec bain amélioré. La différence moyenne entre les types (1) et (2) ou (3) réside dans la rugosité de surface importante, de l'ordre de $1,8 \mu \mathrm{m}$ de (1) comparée à celle de (2) et (3) qui n'est que d'environ $0,1 \mu \mathrm{m}$. Cette différence provient de l'état de surface initial du substrat et n'induit que de faibles variations de $a$ et $e$ (Tableau II).

Le temps de dépôt de $90 \mathrm{~s}$ conduit aux courbes de sélectivité de la figure 8 , avec une fréquence de coupure située entre 4 et $5 \mu \mathrm{m}$. Compte tenu de la pente faible au voisinage de $\lambda_{\mathrm{c}}$, il faut rappeler que $\lambda_{\mathrm{c}}$ est définie par $R\left(\lambda_{\mathrm{c}}\right)=0,50$. Des valeurs de $\lambda_{\mathrm{c}}$ plus faibles peuvent être obtenues avec des temps de dépôt légèrement plus courts.

Tableau II. - Absorption solaire (masse d'air 1) et émissivité infrarouge $\left(T_{\mathrm{a}}=20^{\circ} \mathrm{C}\right)$ de trois échantillons représentatifs des dépôts sur acier nickelé (1), cuivre (2), cuivre nickelé (3).

[Solar absorptance (air-mass 1) and infrared emittance $\left(T_{\mathrm{a}}=20^{\circ} \mathrm{C}\right)$ of 3 samples representing deposits on nickeled steel (1), copper (2), nickeled copper (3).]

$\begin{array}{cccc}\text { Echantillon type } & 1 & 2 & 3 \\ - & - & - & - \\ \text { substrat } & \text { acier nickelé } & \text { cuivre } & \text { cuivre nickelé } \\ a(\%) & 95,5 & 97,0 & 96,8 \\ e(\%) & 13,4 & 9,5 & 14,0\end{array}$




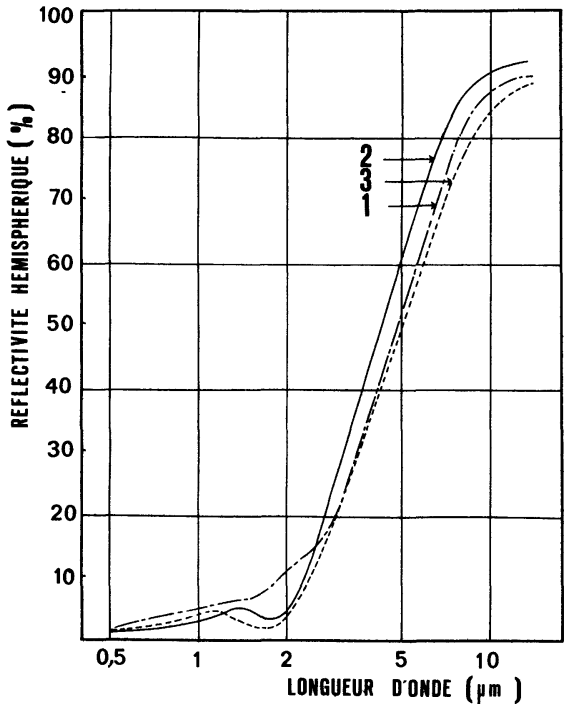

Fig. 8. - Réflectivité hémisphérique des échantillons-types de 0,5 à $15 \mu \mathrm{m}$ sous incidence voisine de la normale.

[Hemispherical reflectivity of mean samples from 0.5 to $15 \mu \mathrm{m}$ under near normal incidence.]

3.3 VARIATIONS DE LA RÉFLECTIVITÉ HÉMISPHÉRIQUE AVEC L'INCIDENCE. - Les courbes de la figure 9 présentent la réflectivité hémisphérique des échantillons (1), (2), (3) mesurée en fonction de l'angle d'incidence et de l'état de polarisation de la radiation incidente pour les longueurs d'onde 0,6 et $1,5 \mu \mathrm{m}$. L'effet de l'incidence ne devient notable qu'au-delà de $40^{\circ}$, il conduit à une augmentation de la réflectivité du revêtement qui peut être multipliée par 5 entre la normale et $70^{\circ}$. Ce fait doit être pris en considération dans le cas de capteurs recevant le flux solaire sous incidence variable. L'effet de l'état de polarisation de la lumière (parallèlement ou perpendiculairement au plan d'incidence) est lui aussi important aux fortes incidences. Compte tenu de la polarisation non négligeable introduite par les optiques de concentration, cette sensibilité de la sélectivité à la polarisation du rayonnement incident peut être ainsi exploitée au bénéfice du rendement du capteur. Notons que l'effet de dépolarisation dû à la rugosité de surface et se traduisant par une relative égalisation des valeurs de $R_{\mathrm{p}}$ et $R_{\mathrm{s}}$ est maximum aux longueurs d'onde voisines des dimensions caractéristiques de la rugosité (courbe 1 de la figure $9 b$, rugosité de $1,8 \mu \mathrm{m}$ à $\lambda=1,5 \mu \mathrm{m}$ ).

3.4 Diffusion DE LA Lumière. - Compte tenu de leur faible rugosité, les échantillons (2) et (3) sont peu diffusants. Par contre, l'échantillon (1) déposé sur acier nickelé présentant une rugosité d'environ $1,8 \mu \mathrm{m}$ diffuse notablement la lumière. Les courbes de diffusion autour de la direction de réflexion spéculaire mesurées à 2,3 et $10 \mu \mathrm{m}$ pour les incidences de 20 à $75^{\circ}$ (Fig. 10) permettent de voir que la diffusion importante à $2,3 \mu \mathrm{m}$, à faible incidence, décroît ensuite avec l'incidence et la longueur d'onde. Autrement dit, à $10 \mu \mathrm{m}$ et à $75^{\circ}$ d'incidence, malgré la rugosité, les surfaces ont un comportement quasi spéculaire.
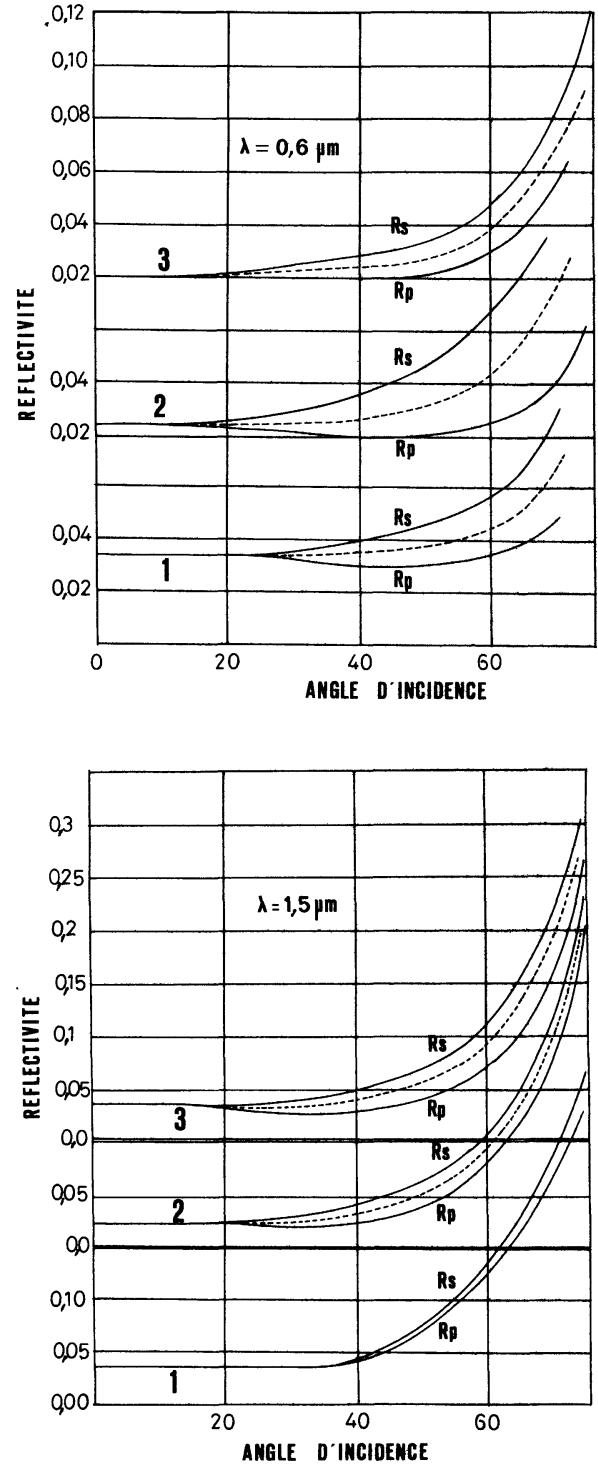

Fig. 9. - Réflectivité hémisphérıque des échantillons (1), (2), (3) en fonction de l'angle d'incidence et de l'état de polarisation de la radiation incidente $\left.\left.\left(R_{\mathrm{p}}, R_{\mathrm{s}}, \frac{R_{\mathrm{p}}+R_{\mathrm{s}}}{2}\right) .9 a\right) \lambda=0,6 \mu \mathrm{m} ; 9 b\right)$ $\lambda=1,5 \mu \mathrm{m}$.

[ Polarized hemispherical reflectivity $\left(R_{\mathrm{p}}, R_{\triangleleft}, \frac{R_{\mathrm{p}}+R_{4}}{2}\right)$ of samples (1), (2), (3) versus angle of incidence ( $Y$ axis translated). 9a) $\lambda=0,6 \mu \mathrm{m} ; 9 b) \lambda=1.5 \mu \mathrm{m}$.]

4. Conclusion. - Nous avons calculé le gain apporté par les surfaces sélectives dans les applications aux capteurs photothermiques à moyenne température (jusqu'à $250^{\circ} \mathrm{C}$ ). Nous avons montré, en détaillant ses propriétés optiques et sa stabilité, que le Chrome Noir était un bon matériau sélectif particulièrement dans ce domaine de températures, dans le cas des concentrations faibles ou moyennes ou des flux solaires moyens. Nous avons fourni tous les éléments permettant d'évaluer le bilan thermique d'une surface de Chrome Noir travaillant sous incidence non normale (jusqu'à $75^{\circ}$ ), éventuellement sous flux incident polarisé. 

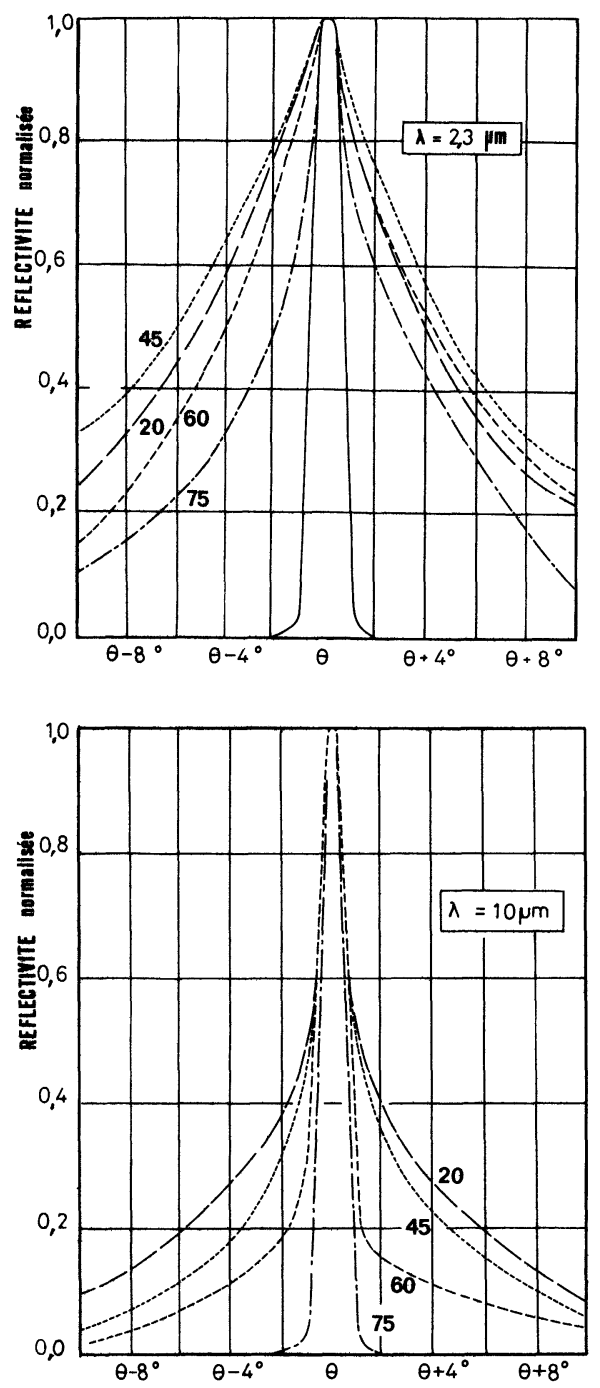

Au-delà de $300^{\circ} \mathrm{C}$ le Chrome Noir n'est plus stable et jusqu'à présent aucun matériau sélectif comparable, par ses performances, n'est venu prendre le relais. Or la sélectivité du Chrome Noir résulte [6] de l'association au sein d'un même composé d'un revêtement de surface antireflet et d'un matériau composite avec inclusions métalliques, dont la partie à faible densité en métal constitue l'absorbant solaire et la partie à forte densité en métal le réflecteur infrarouge. La transposition d'une telle association avec des matériaux différents, comme un métal peu oxydable et une matrice réfractaire, pourrait constituer une bonne surface sélective pour les températures supérieures à $250^{\circ} \mathrm{C}$.

Remerciements. - Nous tenons à remercier J. Spitz, A. Aubert et J. Valignat du Département de Métallurgie du C.E.N.G. pour leur fructueuse et sympathique collaboration à ce travail, notamment l'élaboration des échantillons et l'étude de leur stabilité.

Fig. 10. - Dispersion angulaire de la réflectivité normalisée de l'échantillon (1) à $\theta=20^{\circ}(--\longrightarrow), 45^{\circ}(\ldots), 60^{\circ}(----), 75^{\circ}$ $((-. \cdot-)$, facteur d'appareil (-). 10a) $\lambda=2,33 \mu \mathrm{m} ; 10 b)$ $\lambda=10,5 \mu \mathrm{m}$.

[Angular dispersion of normalized reflectivity of sample (1) at $\theta=20^{\circ}(--\longrightarrow), 45^{\circ}(\ldots), 60^{\circ}(-----), 75^{\circ}(-\cdot-\cdot-)$, instrument function (-). 10a) $\lambda=2.33 \mu \mathrm{m} ; 10 b) \lambda=10.5 \mu \mathrm{m}$.]

\section{Bibliographie}

[1] Spitz, J., Aubert, A., Behaghel, J. M., Berthier, S., Lafait, J., Rivory, J., Revue Phys. Appl. 14 (1979) 67.

[2] DrIVER, P. M., in sun II, proceedings of ISES Conference, Atlanta 1979. K. W. Boër, B. H. Glenn editors (Pergamon Press-New York) 1979, p. 1887.

[3] Spitz, J., Danh, T. V., AuberT, A., Sol. Energy Mater. 1 (1979) 189.
[4] Lafait, J., Berthier, S., Behaghel, J. M., in sun II, proceedings of ISES Conference, Atlanta 1979. K. W. Boër, B. H. Glenn editors (Pergamon Press-New York) 1979, p. 1922.

[5] Behaghel, J. M., Berthier, S., Lafait, J., Rivory, J., Sol. Energy Mater. 1 (1979) 201.

[6] Berthier, S., Lafait, J., J. Physique 40 (1979) 1093. 